\title{
殼の一般的降伏条件についで
}

正会員横 尾義 貫 ${ }^{* *}$ 同松 岡 理 ${ }^{* * *}$

I. 序説 殸の微小要素の降伏条件はこれまですべて 軸対称の場合飞ついての及論ぜられて来たようである。 Onat と Prager ${ }^{(4)}$ とよつて導かれた Tresca の降伏条 件汇従う剛塑性回転款の軸対 称 問 題のための 降伏条件 は、媒介変数表示であるが、それるで導かれたいくつ かの特殊な場合 ${ }^{(1)(2)(3)}$ をすへて含む一般的なるのであ る。この降代条件は $E^{4}$ 空間の中の hypersurface であ つて彼等によつて示されたように、座標平面によるその 断面怙々の特殊な場合に対する降伏曲線を与兄る。 この hypersurface はいくつかの特異な部分を持ち、又 媒介変数表示であることから、実際に種々の殼の終局荷 重の下界を求めるのには多小不便であるが、上界は若干 の計算を遂行すれば容易に求まる。

本論文では更飞一般的な場合飞対する降伏条件を導 く。即殼が von Mises の降伏条件飞従う剛塑性材料で つくられ、plastic potential は乞の降伏函数汇一致する ものとする。この仮定の下に Limit Analysis'の定理 ${ }^{(5)}$ を用いて、一般の非軸対称問題の場合汇対する降伏条件 を導く。やはり媒介変数表示ではあるが、各種の殸の極 限荷重の少くとも上界を求めることは、初期塑性流れの 速度場を適当汇選えで、やや煩雑な計算を遂行すれば可 能であろう。

次にこの一般媒介変数表示から、一つの特殊な場合と 乙て床板の降伏条件を直ち導く。

更に圧縮及び弓張降伏応力が異るような、Tresca 型 の条件良う剛塑性材料で作られている殼江対する降伏 条件を、軸対称問題の場合飞ついて、Onat ${ }^{(3)}$ の方法を 拡張して媒介变数表示で導き、これがその一つの特殊な 場合として、通常の Tresca 材料沉つて彼が既汇導い た結果をる含むやや一般的なるのであることを示す。

最後に上の一般降伏条件が奏際江用いるのと相当不便 であることを考兄、極限荷重の概算の為汇若干の近似式 をやや直観的ではあるがここに提案する。

\section{II. 一般降伏条件}

$\$ 2.1$ Lower Bound of Yield Hypersurface

殼の微小要素の各層の降伏状態を論ずるに考慮すべき 応力は、第 1 図のように( $\alpha_{z}$ 及び $\tau_{\beta} z$ の降伏兄の影響

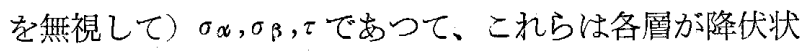

* Limit Analysis of Shells I

** 京都大学教授 (防災研究所) 工博

*** 京都工芸繊維大学助教授

*关兑京都大学大学院生

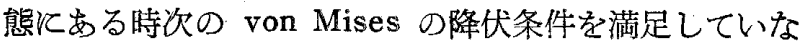
ければならない。

$$
f\left(\sigma_{\alpha}, \sigma_{\beta}, \tau\right)=\sigma_{\alpha}{ }^{2}-\sigma_{\alpha} \sigma_{\beta}+\sigma_{\beta^{2}}+3 \tau^{2}=3 k^{2} \cdots(2.1 .1)
$$

第2 図はその主応力平面上の表示である。さはこの時、 $\sigma_{\alpha}, \sigma_{\beta}$ で表わされ

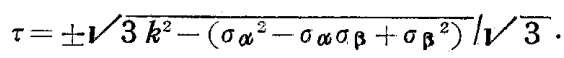

かような全塑性状態㳊るる殼の微小要素作用している

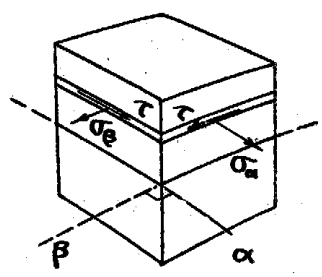

第 1 図

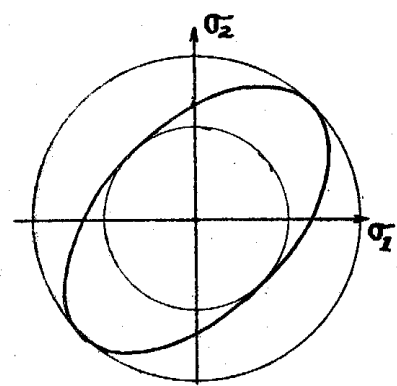

第 2 図
断面力は通常の曲面板理論で次式で与穴られている。

$$
\left.\begin{array}{c}
N_{i}=\int_{-h / 2}^{h / 2} \sigma_{i} d z \quad M_{i}=\int_{-h / 2}^{h / 2} \sigma_{i} z d z \quad(i=\alpha, \beta) \\
S= \pm \frac{1}{\sqrt{3}} \int_{-h / 2}^{h / 2} \sqrt{3 k^{2}-\left(\sigma_{\alpha^{2}}-\sigma_{\alpha} \sigma_{\beta}+\sigma_{\beta}\right)^{2}} d z \\
M_{\alpha \beta}= \pm \frac{1}{\sqrt{3}} \int_{-h / 2}^{h / 2} \sqrt{3 k^{2}-\left(\sigma_{\alpha}{ }^{2}-\sigma_{\alpha} \sigma_{\beta}+\sigma_{\beta}{ }^{2}\right)} z d z
\end{array}\right\}
$$

$N_{\alpha}, N_{\beta}, S ; M_{\alpha}, M_{\beta}, M_{\alpha \beta}$ の作用している殸の微小要 素の降伏条件を求めることは、その各膡で降伏条件 $(2$. 1.1)㝊満足する応力の組 $\left[\sigma_{\alpha}(z), \sigma_{\beta}(z), \tau(z)\right]$ の集合か ら、適当な崩壊断面力の組の集合を算定して、 $E^{6}$ 空間 で原点を含み、convexである筈の真のYield Hypersurface の内部で出来るだけこれと近い Hypersurface $S_{L}{ }^{5}$ を求めることを意味する。これは次のように問題の表現 を変劣れば、一種の等周問題として解くことが出来る。

崩壊断面力の表現 (2.1.3) の 6 個の断面の中の 5 個の 式を附帯条件と見なし、その 5 個の断面力の或組炕対し て残りの 6 番目の断面力を最大沉するような $\sigma_{\alpha}, \sigma_{\beta}$ の

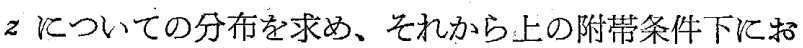
ける 6 番目の断面力の最大值を求めると、ここ得られ た崩溒断面力の一組は下界超曲面 $S_{L}{ }^{5}$ の上の一点を与 える。そこで附带条件として取つた初めの 5 個の断面力 の組の集合のすべてを採れば $S_{L}^{5}$ のすべての点が与えら 㣗得ると考えられる。（例学ば、曲げモーメント $M$ と 据りモメーントT の作用する棒材の場合を想定すれば、 
類推によつてこの表現の妥当性が容易に確認されよう)

問題に対する Euler の方程式は次の有限な形で与えら

Lagrange の乗数を $\alpha_{1}, \alpha_{2}, \beta_{1}, \beta_{2}, \lambda$ で表わせば、この れる。

$$
\begin{aligned}
& \frac{\partial}{\partial \sigma_{i}}\left( \pm \frac{1}{\sqrt{3}} \sqrt{3 k^{2}-\left(\sigma_{\alpha}{ }^{2}-\sigma_{\alpha} \sigma_{\beta}+\sigma_{\beta}{ }^{2}\right)}\right)+\lambda \frac{\partial}{\partial \sigma_{i}}\left( \pm \frac{z}{\sqrt{3}} \sqrt{3 k^{2}-\left(\sigma_{\alpha}{ }^{2}-\sigma_{\alpha \sigma} \sigma_{\beta}+\sigma_{\beta}{ }^{2}\right)}\right) \\
& +\alpha_{1} \frac{\partial}{\partial \sigma_{i}}\left(\sigma_{\alpha}\right)+\alpha_{2} \frac{\partial}{\partial \sigma_{i}}\left(\sigma_{\alpha} z\right)+\beta_{1} \frac{\partial}{\partial \sigma_{i}}\left(\sigma_{\beta}\right)+\beta_{2} \frac{\partial}{\partial \sigma_{i}}\left(\sigma_{\beta} z\right)=0 \quad(i=\alpha, \beta) \cdots \ldots \ldots \ldots
\end{aligned}
$$

$\sigma_{\alpha}, \sigma_{\beta}$ について解けば

$$
\begin{aligned}
& \sigma_{\alpha}= \pm k \frac{2\left(\alpha_{1}+\alpha_{2} z\right)+\left(\beta_{1}+\beta_{2} z\right)}{\sqrt{(1+\lambda z)^{2}+\left(\alpha_{1}+\alpha_{2} z\right)^{2}+\left(\alpha_{1}+\alpha_{2} z\right)\left(\beta_{1}+\beta_{2} z\right)+\left(\beta_{1}+\beta_{2} z\right)^{2}}} \\
& \sigma_{\beta}= \pm k \frac{2\left(\beta_{1}+\beta_{2} z\right)+\left(\alpha_{1}+\alpha_{2} z\right)}{\sqrt{(1+\lambda z)^{2}+\left(\alpha_{1}+\alpha_{2} z\right)^{2}+\left(\alpha_{1}+\alpha_{2} z\right)\left(\beta_{1}+\beta_{2} z\right)+\left(\beta_{1}+\beta_{2} z\right)^{2}}} \\
& \tau= \pm k \frac{1+\lambda z}{\sqrt{(1+\lambda z)^{2}+\left(\alpha_{1}+\alpha_{2} z\right)^{2}+\left(\alpha_{1}+\alpha_{2} z\right)\left(\beta_{1}+\beta_{2} z\right)+\left(\beta_{1}+\beta_{2} z\right)^{2}}} \\
& N_{\alpha}= \pm k \int_{-h / 2}^{h / 2} \frac{\left(D_{\alpha} z+E_{\alpha}\right) d z}{\sqrt{A z^{2}+B z+C}}, \quad M_{\alpha}= \pm k \int_{-h / 2}^{h / 2} \frac{\left(D_{\alpha} z+E_{\alpha}\right) z d z}{\sqrt{A z^{2}+B z+C}} \\
& N_{\beta}= \pm k \int_{-h / 2}^{h / 2} \frac{\left(D_{\beta} z+E_{\beta}\right) d z}{\sqrt{A z^{2}+B z+C}}, \quad M_{\beta}= \pm k \int_{-h / 2}^{h / 2} \frac{\left(D_{\beta} z+E_{\beta}\right) z d z}{\sqrt{A z^{2}+B z+C}} \\
& S= \pm k \int_{-h / 2}^{h / 2} \frac{(1+\lambda z) d z}{\sqrt{A z^{2}+B z+C}} \\
& M_{\alpha \beta}= \pm k \int_{-h / 2}^{h / 2} \frac{(1+\lambda z) z d z}{\sqrt{A z^{2}+B z}+\bar{C}}
\end{aligned}
$$

と打けば崩㳖断面力の組は次のように表わされる。

積分尘行すれば $N_{\alpha}, N_{\beta}, S ; M_{\alpha}, M_{\beta}, M_{\alpha \beta}$ を座標軸 とする $E^{6}$ 空間の申の Lower Bound Yield Hypersurface $S_{L}{ }^{5}$ の媒介変数 $\alpha_{1}, \alpha_{2}, \beta_{1}, \beta_{2}, \lambda$ による表示を得る。

$\S 2.2$. Upper Bound of Yield Hypersurface

Limit Design の一般定理 ${ }^{(5)}$ にれね゙、力学的エネル ギー消散速度は一般応力 $N_{\alpha}, N_{\beta}, S ; M_{\alpha}, M_{\beta}, M_{\alpha \beta}$ 及 びこれに対応する一般歪速度 $\varepsilon_{\alpha}, \varepsilon_{\beta}, r ; \kappa_{\alpha}, \kappa_{\beta}, \theta$ の項で

$$
\begin{aligned}
D & =N_{\alpha} \varepsilon_{\alpha}+N_{\beta} \varepsilon_{\beta}+S \gamma \\
& +M_{\alpha} \kappa_{\alpha}+M_{\beta} \kappa_{\beta}+M_{\alpha \beta} \theta
\end{aligned}
$$

で与えられる。ここに $\varepsilon_{\alpha}, \varepsilon_{\beta}, r$ は夫々中央面の伸び及 び前断歪速度、 $\kappa_{\alpha}, \kappa_{\beta}$ は曲率速度、 $\theta$ 恃振り回転角速度 を表わす。

他方、仮定した材料の非粘性によつて、Dは一般歪速 度の一次同次式で表わされねね゙ならない。従つて同次函 数についての Euler の定理により

$$
\begin{aligned}
D & =\frac{\partial D}{\partial \varepsilon_{\alpha}} \varepsilon_{\alpha}+\frac{\partial D}{\partial \varepsilon_{\beta}} \varepsilon_{\beta}+\frac{\partial D}{\partial \gamma} \gamma \\
& +\frac{\partial D}{\partial \kappa_{\alpha}} \kappa_{\alpha}+\frac{\partial D}{\partial \kappa_{\beta}} \kappa_{\beta}+\frac{\partial D}{\partial \theta} \theta
\end{aligned}
$$

(2.2.1) と (2.2.2) 加占次の関係式老得る。

$$
\begin{aligned}
& N_{\alpha}=\frac{\partial D}{\partial \varepsilon_{\alpha}}, \quad N_{\beta}=\frac{\partial D}{\partial \varepsilon_{\beta}}, \quad S=\frac{\partial D}{\partial \gamma} \\
& \left.M_{\alpha}=\frac{\partial D}{\partial \kappa_{\alpha}}, \quad M_{\beta}=\frac{\partial D}{\partial \kappa_{\beta}}, \quad M_{\alpha \beta}=\frac{\partial D}{\partial \theta}\right\} \\
& D=\int_{-h / 2}^{h / 2} 2 k \sqrt{e_{\alpha}^{2}+e_{\alpha} e_{\beta}+e_{\beta}{ }^{2}+\left(\frac{\gamma \alpha \beta}{2}\right)^{2}} d z \\
& =\int_{-h / 2}^{h / 2} 2 k \sqrt{\left(\varepsilon_{\alpha}+\kappa_{\alpha} z\right)^{2}+\left(\varepsilon_{\alpha}+\kappa_{\alpha} z\right)\left(\varepsilon_{\beta}+\kappa_{\beta} z\right)+\left(\varepsilon_{\beta}+\kappa_{\beta} z\right)^{2}+\frac{1}{4}(\gamma+\theta z)^{2}} \text {. }
\end{aligned}
$$

このDを(2.2.3) 式に代入すれね゙

$$
N_{\alpha}= \pm k \int_{-h / 2}^{h / 2} \frac{\left\{2\left(\varepsilon_{\alpha}+\kappa_{\alpha} z\right)+\left(\varepsilon_{\beta}+\kappa_{\beta} z\right)\right\} d z}{\sqrt{\left(\varepsilon_{\alpha}+\kappa_{\alpha} z\right)^{2}+\left(\varepsilon_{\alpha}+\kappa_{\alpha} z\right)\left(\varepsilon_{\beta}+\kappa_{\beta} z\right)+\left(\varepsilon_{\beta}+\kappa_{\beta} z\right)^{2}+(\gamma+\theta z)^{2} / 4}}
$$

上界超曲面 $S_{U}{ }^{5}$ 上の点を与える崩壊断面力の組 $\left[N_{\alpha}\right.$ ， $\left.N_{\beta}, S ; M_{\alpha}, M_{\beta}, M_{\alpha \beta}\right]$ を得るためには、まず殸の微小 要素の初期塑性流れの際の $D$ を計算せねなない、そ こでこの初期塑性流れの速度場として、次のような曲げ と捱れの合成されたものを考觉る。

$e_{\alpha}=\varepsilon_{\alpha}+\kappa_{\alpha} z, e_{\beta}=\varepsilon_{\beta}+\kappa_{\beta} z, \gamma_{\alpha \beta}=\gamma+\theta z \cdots(2.2 .4)$

ここに $e_{\alpha}, e_{\beta}, \gamma_{\alpha \beta}$ は各層の塑性歪速度を表わす。この 場合の $D$ は次のように書ける。

$$
D=\int_{-h / 2}^{h / 2}\left(\sigma_{\alpha} e_{\alpha}+\sigma_{\beta} e_{\beta}+\tau \gamma_{\alpha \beta}\right) d z
$$

plastic potentialが仮定した降伏函数に一致するものと 仮定したから、応力と昰速度の間には次式が成立つ。

$$
e_{\alpha}=\mu \frac{\partial f}{\partial \sigma_{\alpha}}, \quad e_{\beta}=\mu \frac{\partial f}{\partial \sigma_{\beta}}, r_{\alpha \beta}=\mu \frac{\partial f}{\partial \tau}
$$

ここに $\mu$ は正の乗数で、 $f$ は (2.1.1) 式の $f\left(\sigma_{\alpha}, \sigma_{\beta}, \tau\right)$ だから $e_{\alpha}=\mu\left(2 \sigma_{\alpha}-\sigma_{\beta}\right), e_{\beta}=\mu\left(2 \sigma_{\beta}-\sigma_{\alpha}\right)$,

$$
\gamma_{\alpha \beta}=6 \mu \tau
$$

仅定した歪速度に対応する応力が、煌含んで $e_{\alpha}, e_{\beta}$ ，

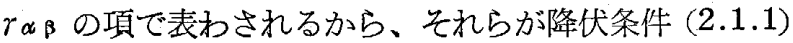
式を満足するようと同式代入して れらの応力は $e_{\alpha}, e_{\beta}, r \alpha \beta$ のの項で表わすことが出来 る。従つて $D$ 的 $e_{\alpha}, e_{\beta}, \gamma \alpha \beta$ のによつて次のように表 


$$
\begin{aligned}
N_{\beta} & = \pm k \int_{-h / 2}^{h / 2} \frac{\left\{2\left(\varepsilon_{\alpha}+\kappa_{\alpha} z\right)+\left(\varepsilon_{\beta}+\kappa_{\beta} z\right)\right\} d z}{\sqrt{\left(\varepsilon_{\alpha}+\kappa_{\alpha} z\right)^{2}+\left(\varepsilon_{\alpha}+\kappa_{\alpha} z\right)\left(\varepsilon_{\beta}+\kappa_{\beta}^{\prime \prime} z\right)+\left(\varepsilon_{\beta}+\kappa_{\beta} z\right)^{2}+(\gamma+\theta z)^{2} / 4}} \\
S & = \pm k \int_{-h / 2}^{h / 2} \sqrt{\frac{\{(\gamma+\theta z) / 2\} d z}{\sqrt{\left(\varepsilon_{\alpha}+\kappa_{\alpha} z\right)^{2}+\left(\varepsilon_{\alpha}+\kappa_{\alpha} z\right)\left(\varepsilon_{\beta}+\kappa_{\beta} z\right)+\left(\varepsilon_{\beta}+\kappa_{\beta} z\right)^{2}+(\gamma+\theta z)^{2} / 4}}} \\
M_{\alpha} & = \pm k \int_{-h / 2}^{h / 2} \frac{\left\{2\left(\varepsilon_{\alpha}+\kappa_{\alpha} z\right)+\left(\varepsilon_{\beta}+\kappa_{\beta} z\right)\right\} z d z}{\sqrt{\left(\varepsilon_{\alpha}+\kappa_{\alpha} z\right)^{2}+\left(\varepsilon_{\alpha}+\kappa_{\alpha} z\right)\left(\varepsilon_{\beta}+\varepsilon_{\beta} z\right)+\left(\varepsilon_{\beta}+\kappa_{\beta} z\right)^{2}+(\gamma+\theta z)^{2} / 4}} \\
M_{\beta} & = \pm k \int_{-h / 2}^{h / 2} \frac{\left\{2\left(\varepsilon_{\beta}+\kappa_{\beta} z\right)+\left(\varepsilon_{\alpha}+\kappa_{\alpha} z\right)\right\} z d z}{\sqrt{\left(\varepsilon_{\alpha}+\kappa_{\alpha} z\right)^{2}+\left(\varepsilon_{\alpha}+\kappa_{\alpha} z\right)\left(\varepsilon_{\beta}+\kappa_{\beta} z\right)+\left(\varepsilon_{\beta}+\kappa_{\beta} z\right)^{2}+(\gamma+\theta z)^{2} / 4}} \\
M_{\alpha \beta} & = \pm k \int_{-h / 2}^{h / 2} \frac{\{(\gamma+\theta z) / 2\} z d z}{\sqrt{\left(\varepsilon_{\alpha}+\kappa_{\alpha} z\right)^{2}+\left(\varepsilon_{\alpha}+\kappa_{\alpha} z\right)\left(\varepsilon_{\beta}+\kappa_{\beta} z\right)+\left(\varepsilon_{\beta}+\kappa_{\beta} z\right)^{2}+(\gamma+\theta \boldsymbol{z})^{2} / 4}}
\end{aligned}
$$

(2.2.8)式で $\varepsilon_{\alpha}, \varepsilon_{\beta}, \gamma ; \kappa_{\alpha}, \kappa_{\beta}, \theta$ の 6 個対してその

間の 5 個の比を与杂标、 $N_{\alpha}, N_{\beta}, S ; M_{\alpha}, M_{\beta}, M_{\alpha \beta}$ の 值が確定するから今とれらの比を

$\frac{\theta}{r} \equiv \lambda, \frac{2 \varepsilon_{\alpha}}{r} \equiv \alpha_{1}, \quad \frac{2 \kappa_{\alpha}}{r} \equiv \beta_{2}, \frac{2 \varepsilon_{\beta}}{r} \equiv \beta_{1}, \quad \frac{2 \kappa_{\beta}}{r} \equiv \beta_{2}$ と括けば、(2.2.8) 式の表現は全く.(2.1.6) 式に一致す る。従つてこの媒介変数表示の超曲面は、仮定の制限内 そ括いて真の Yield Hypersurface である。

\section{$\$ 2.3$. Parametric Representation of the Yield}

Hypersurface \& the associated flow rule.

無次元でまとまつた形で表示するためと次のような新 しい記号を用いる。

$$
\begin{aligned}
& \frac{N_{\alpha}}{N_{0}}= \pm \frac{1}{\sqrt{3}}\left[\frac{a_{\alpha} c}{p}+\frac{2 b_{\alpha} p-a_{\alpha} q}{2 p \sqrt{p}} l\right] \frac{N_{\beta}}{N_{0}}= \pm \frac{1}{\sqrt{3}}\left[\frac{a_{\beta} c}{p}+\frac{2 b_{\beta} p-a_{\beta} q}{2 p \sqrt{p}}\right] l \\
& \frac{S}{N_{0}}= \pm \frac{1}{\sqrt{3}}\left[\frac{\nu c}{p}+\frac{2 p-\nu q}{2 p \sqrt{p}} \cdot l\right] \\
& \frac{M_{\alpha}}{M_{0}}= \pm \frac{1}{\sqrt{3}}\left[\frac{4 b_{x} p-3 a_{\alpha} q}{p} \cdot c+\frac{2 a_{\alpha} d}{p}+\frac{\left(3 a_{\alpha} q-4 b_{\alpha} p\right) q-4 a_{\alpha} p r}{2 p \sqrt{p}} \cdot l\right] \\
& \frac{M_{\beta}}{M_{0}}= \pm \frac{1}{\sqrt{3}}\left[\frac{4 b_{\beta} p-3 a_{\beta} q}{p} \cdot c+\frac{2 a_{\beta} d}{p}+\frac{\left(3 a_{\beta} q-4 b_{\beta} p\right) q-4 a_{\beta} p r}{2 p \sqrt{p}} \cdot l\right] \\
& \frac{M_{\alpha \beta}}{M_{0}}= \pm \frac{1}{\sqrt{3}}\left[\frac{4 p-3 \nu q}{p} \cdot c+\frac{2 \nu d}{p}+\frac{(3 \nu q-4 p) q-4 \nu p r}{2 p \sqrt{p}} \cdot l\right]
\end{aligned}
$$

これ対応する flow rule は

$N_{0} \varepsilon_{\alpha}: N_{0} \varepsilon_{\beta}: N_{0} \tau: M_{0} \kappa_{\alpha}: M_{0} \kappa_{\beta}: M_{0} \theta$ $=2 \alpha: 2 \beta: 1: 8 \alpha^{\prime}: 8 \beta^{\prime}: 4 \nu$

$\$ 2.4$ 平板の降伏条件

この場合には (2.2.8) 式で $\varepsilon_{\alpha} \equiv \varepsilon_{\beta} \equiv \gamma \equiv 0$ と和忛るから、新しい曲率速度の比

$$
\nu_{\alpha}=\frac{2 \kappa_{\alpha}}{\theta}, \quad \nu_{\beta}=\frac{2 \kappa_{\beta}}{\theta}
$$

を用いて次のような簡単な形になる。

$$
\begin{aligned}
& M_{\alpha}= \pm \frac{M_{0}}{\sqrt{3}} \frac{2 \nu_{\alpha}+\nu_{\beta}}{\sqrt{1+\left(\nu_{\alpha}^{2}+\nu_{\alpha} \nu_{\beta}+\nu_{\beta}^{2}\right)}} \\
& M_{\beta}= \pm \frac{M_{0}}{\sqrt{3}} \frac{2 \nu_{\beta}+\nu_{\alpha}}{\sqrt{1+\left(\nu_{\alpha}^{2}+\nu_{\alpha} \nu_{\beta}+\nu_{\beta}^{2}\right)}} \\
& M_{\alpha \beta}= \pm \frac{M_{0}}{\sqrt{3}} \frac{1}{\sqrt{1+\left(\nu_{\alpha}^{2}+\nu_{\alpha} \nu_{\beta}+\nu_{\beta}^{2}\right)}}
\end{aligned}
$$

この場合には、 $\nu_{\alpha}, \nu_{\beta}$ は容易消消去でさて

$$
M_{\alpha}{ }^{2}-M_{\alpha} M_{\beta}+M_{\beta}{ }^{2}+3 M_{\alpha \beta}{ }^{2}=M_{0}{ }^{2}
$$

III. Modified Tresca Condition に従う剛塑性回

\section{転殼の軸対称問題のための降伏条件}

款の材料が、建築物としては主として鉄筋コンクリー トであることを考虑し、以下、は材料が第 3 四の如き Modified Tresca Condition に徒う岡㨫性材料であると して、その軸対称問題のための殼要素の降伏条件を導い

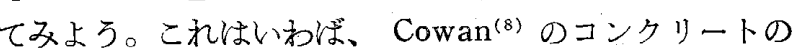
破壊曲面を降伏曲面として持つような材料、或は Drucker 及び Shield ${ }^{(6)(7)}$ そよつて上に対する完全塑性理論 の適用のため提出された降伏曲面核従う材料を想定し たことを意味する。ここ示す方法は、Onat が円筒の 場合飞採用した手法 ${ }^{(3)}$ を一般軸対称問題の場合飞拡張し たるのである。従つてここに導かれる降伏条件は材料の 引張降伏応力の変化を規定する parameter を大れた多 小一般化されたもので Onat とPrager が導いた結果 ${ }^{(4)}$ をも含さすのである。

殼の微小要素が降伏状態年る時、各首の応力状態は 第3図の Modified Tresca Condition とそれに対応する flow rule を満足している。ここでは軸対称の場合に限 るから、各層の塑性主歪速度の比は

$$
\frac{e_{\varphi}}{e_{\theta}}=\frac{\kappa_{\varphi}}{\kappa_{\theta}} \cdot \frac{\varepsilon_{\varphi} / \kappa_{\varphi}-z}{\varepsilon_{\theta} / \kappa_{\theta}-z}=\alpha \frac{\varepsilon_{\varphi} / \kappa_{\varphi}-z}{\varepsilon_{\theta} / \kappa_{\theta}-z}=\cot \nu, \alpha \equiv \frac{\kappa_{\varphi}}{\kappa_{\theta}}
$$
と表わされ、独立な三変量 $\alpha, \varepsilon_{\varphi} / \kappa_{\varphi}, \varepsilon_{\theta} / \kappa_{\theta}$ Kよつてv が定まるか、各層の歪速度べクトルの方向が定まる。 従つてこれに対応する応力状態は第 3 図を考慮すれば第 4 図下方に示すように一意に定末る。従つて $\left[\alpha, \varepsilon \varphi / \kappa_{\varphi}\right.$, $\left.\varepsilon_{\theta} / \kappa_{\theta}\right]$ の或值の組に対してこれに対応する断面力の絹が 
算定される。この手続を $\alpha, \varepsilon_{\varphi} / \kappa_{\varphi}, \varepsilon_{\theta} / \kappa_{\theta}$ のすべての領 域にわたつて行兄ば、図中の新しい parameters $p, q, r$, $s$ 孝用いて、第 1 表の如く降伏条件の媒介变数表示を得 る。明らか汇 $\lambda=1$ と和けば、Onat \& Pragerの結果 ${ }^{(3)}$ 飞一致する。向 $p, q, r, s$ の 個の中 3 個が独立で、 $\lambda=$ 1 の時 $s=q$ である。注意すべきは表中降伏条件が $p, q$, $r, s$ の順序のみ関係することである。各種の特殊な場 合は夫々誘導され得るが省略する。

対応するflow rule は次式で与えられる。

$$
\begin{aligned}
N_{0} \varepsilon_{\theta} & : N_{0} \varepsilon_{\varphi}: M_{0} \kappa_{\theta}: M_{0} \kappa_{\varphi} \\
\quad= & 4 p(q-r): 4 \lambda r(p-q): q-r: \lambda(p-q)
\end{aligned}
$$

IV. 近似降伏条件

ここに導いた夫々の材料の殼の降伏条件は、いづれる 媒介変数表示であつて使用上相当の不便が予想される。 そこで概算用として、ここに直観的ではあるが次のよう な近似降伏条件—explicit な Yield Hypersurface を 提案する。その一つは

$$
\begin{gathered}
\left(\frac{N_{\alpha}}{N_{0}}\right)^{2}-\left(\frac{N_{\alpha}}{N_{0}}\right)\left(\frac{N_{\beta}}{N_{0}}\right)+\left(\frac{N_{\beta}}{N_{0}}\right)^{2}+3\left(\frac{S}{N_{0}}\right)^{2} \\
+\left(\frac{M_{\alpha}}{M_{0}}\right)^{2}-\left(\frac{M_{\alpha}}{M_{0}}\right)\left(\frac{M_{\beta}}{M_{0}}\right)+\left(\frac{M_{\beta}}{M_{0}}\right)^{2}+3\left(\frac{M_{\alpha \beta}}{M_{0}}\right)^{2}=1
\end{gathered}
$$

であつて、これは $N$ の系と $M$ の系とついては真の降 伏超曲面の断面に一致するが、両者が作用している時例 壳ば

$$
\left(N_{\alpha} / N_{0}\right)^{2}+\left(M_{\alpha} / M_{0}\right)=1
$$

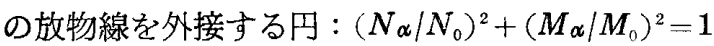

で近似したことを意味するるのである。

$$
\text { 他の一つ站更に大胆飞 }
$$

$$
\begin{aligned}
& \left(\frac{N_{\alpha}}{N_{0}}\right)^{2}+\left(\frac{N_{\beta}}{N_{0}}\right)^{2}+\left(\frac{S}{S_{0}}\right)^{2} \\
& \quad+\left(\frac{M_{\alpha}}{M_{0}}\right)^{2}+\left(\frac{M_{\beta}}{M_{0}}\right)^{2}+\left(\frac{M_{\alpha \beta}}{M_{\alpha \beta_{0}}}\right)^{2}=g^{2} \text { (const) }
\end{aligned}
$$

但し $g$ として例总注主応力平面上の Mises 曲線沈内接 及び外接する円の半径の値をとる。このように真の降伏 超曲面认内接的及び外接的な $S_{L}{ }^{5}$ 之 $S_{U}{ }^{5}$ とを、極限荷 重の下界を求める時には $S_{L}{ }^{5}$ を、上界には $S_{U}{ }^{5}$ を採用 して、その概略の上下界を抑光ようとするるのである。

V. 結論 1. 殼の一般的降伏条件を媒介変数で表示

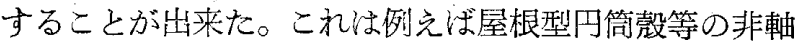
対称問題にも適用できるもので、今後種々の壳について 終局荷重を求方兮際の基礎式が確立できた。

2. II 亿採用した方法はいかなる smooth な降伏曲線

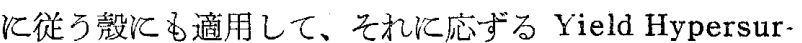
face 求めることが出来る。

3.平板の降伏条件を一般式から容易に導いた。

4. 材料の圧縮及び引張降伏応力の異る場合飞対して

\begin{tabular}{|c|c|c|c|c|}
\hline TABLE & \multicolumn{4}{|c|}{ Dimenstomless Stress Resultants } \\
\hline gronz & $n_{\theta}$ & $n$ & $m$ & \\
\hline & $x_{2} \frac{1}{2}$ & $\lambda\left(\frac{1}{2}+s\right)-\left(\frac{1}{2}-r\right)$ & $2\left(\frac{1}{4}=\right.$ & $22\left(4-s^{3}\right)+2\left(\frac{1}{4}+n^{2}\right.$ \\
\hline$r>p>q$ & $x\left(\frac{1}{2} \cdot 7\right)-\left(\frac{1}{2}-7\right)$ & $-(r-q)$ & $2 x\left(\frac{1}{4}-f^{2}\right)+2\left(\frac{1}{4}-7\right)$ & $z\left(r^{2}-q^{2}\right)$ \\
\hline & $\left.-\frac{1}{2}-p\right)$ & $-\left(\frac{1}{2}+r\right)$ & $2\left(\frac{1}{4}-p^{2}\right)$ & $2\left(r^{2}-\frac{1}{4}\right)$ \\
\hline$s>r>P$ & $-(s-\mu)$ & $\lambda\left(\frac{1}{2}-s\right)-\left(\frac{1}{2}+r\right)$ & $2\left(5^{2}-4^{3}\right)$ & $(-2)\left(1-s^{2}\right)+2\left(t^{\prime}-r^{2}\right)$ \\
\hline & $\left(\frac{1}{2}+8\right)-\left(\frac{1}{2}-r\right)$ & $\lambda\left(\frac{1}{2}+r\right)\left(\frac{1}{2}-x\right)$ & $2 x\left(x^{\prime}-y^{2}\right)+2\left(x^{\prime}-p^{3}\right)$ & $(2)(t+)^{2}+2(1-8)$ \\
\hline & $\lambda\left(r+\frac{1}{2}\right)-\left(\frac{1}{2}-s\right)$ & $\lambda(s-r)$ & $2\left(p^{2}-\frac{1}{4}\right)+2\left(\frac{1}{4}-5\right)$ & $-2 \lambda\left(s^{2}=r^{2}\right)$ \\
\hline & $\lambda(p-q)-\left(\frac{1}{2}-s\right)$ & $A(s-r)-r_{2}^{\prime}$, & $2\left(\theta^{2}-7^{2}\right)$ & $2\left(1 / 2-x^{2}+2\left(\frac{1}{1}-5\right)\right.$ \\
\hline$p>r>q$ & $\lambda(p-q)$ & $\lambda\left(\frac{1}{2}-r\right)-\left(\frac{1}{2}+q\right)$ & $\lambda\left(p^{2}-q^{2}\right)$ & {$\left[E \times\left(\frac{1}{1}+r^{3}+2\left(\frac{1}{1}-1\right)\right.\right.$} \\
\hline
\end{tabular}
軸対称哃題に限つたが、その降伏条件学導くことができ た。これ深气の特殊の場合として、Onat \& Prager の結 果を含み、材料の降伏応力規定寸る parameter を入 れた意味でやや一般的なるので岕る。

5. 極限荷重の上下界の概算のために Yield Hyper-

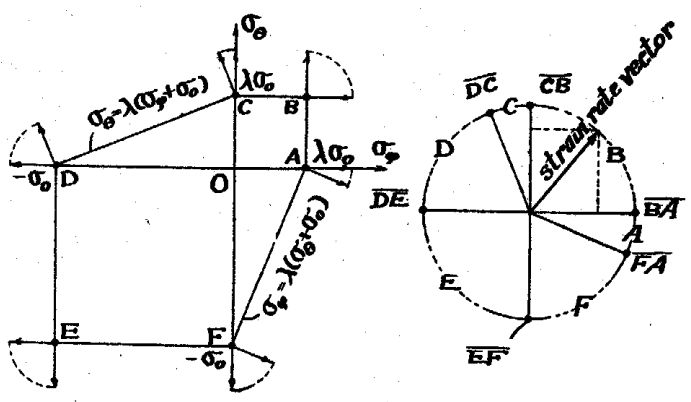

第 3 図

第 1 図

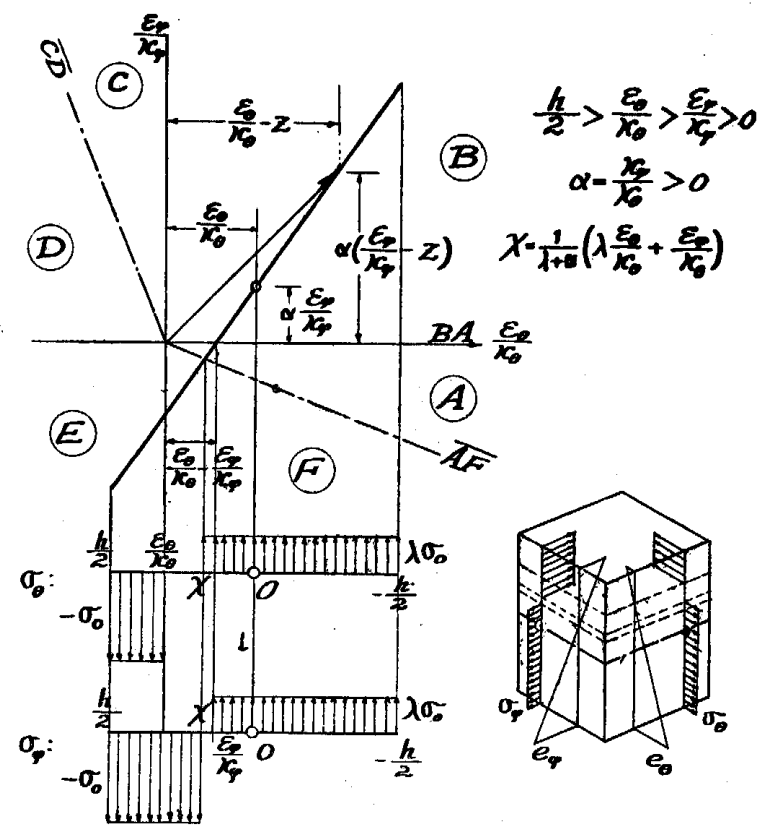

第 4 図

surface の近似上下界超曲面呆提案した。

\section{Bibliography}

(1) D.C. Drucker, Pr. 1st MW Conf. Solid Mech.

(2) P.G. Hodge, Jr., J. Appl. Mech. Dec. 1954

(3) E.T. Onat, J. Appl. Math. 1955

(4) E.T. Onat, \& W. Prager, Koninkl. Nederl. Akademie van Wetenschappen 57. 1954

(5) W. Prager, Proc. 8 Int. Congr. Appl. Mech.

(6) D.C. Drucker, J. Mech. Phys. Solids, 1, 1953

(7) R.T. Shield, J. Mech. Phys. Solids, 4, 1955

(8) H.J. Cowan, Mag. of Conc. Res. 1953

(9) R.T. Shield, D.C. Drucker, Feb. 1958. Brown U.

(10) P.G. Hodge, Jr., J. Appl. Mech. Sept. 1957 